\title{
Micromechanical Uncooled Photon Detectors
}

\author{
Panos G. Datskos a,b \\ aOOak Ridge National Laboratory, MS-8039, Oak Ridge, TN 37831-8039 \\ bUniversity of Tennessee, Department of Physics and Astronomy, Knoxville, TN 37996-1200
}

\begin{abstract}
Recent advances in micro-electro-mechanical systems (MEMS) have led to the development of uncooled infrared detectors operate as micromechanical thermal detectors or micromechanical quantum detectors. We report on a new method for photon detection using electronic (photo-induced) stresses in semiconductor microstructures. Photo-induced stress in semiconductor microstructures, is caused by changes in the charge carrier density in the conduction band and photon detection results from the measurement of the photo-induced bending of semiconductor microstructures. Small changes in position (displacement) of microstructures are routinely measured in atomic force microscopy (AFM) where atomic imaging of surfaces relies on the measurement of small changes $\left(<10^{-9} \mathrm{~m}\right)$ in the bending of microcantilevers. Changes in the conduction band charge carrier density can result either from direct photo-generation of free charge carriers (electrons, holes) or from photoelectrons emitted from thin metal film surfaces in contact with a semiconductor microstructure which forms a Schottky barrier. In our studies we investigated three systems: (i) Si microstructures, (ii) $\mathrm{InSb}$ microstructures and (iii) $\mathrm{Si}$ microstructures coated with a thin film of Pt. We found that for $\mathrm{Si}$ the photo-induced stress results in a contraction of the crystal lattice due to the presence of excess electron-hole-pairs while for InSb photo-induced stress causes the crystal lattice to expand. We will present our results and discuss our findings.
\end{abstract}

Keywords: MEMS, micromechanical photon detector, thermal detector, photo-induced stress

\section{INTRODUCTION}

Photon detection and imaging has extensive medical, industrial, military, and commercial applications. The detection of infrared (IR) radiation, which is the second most intense radiation source in our environment, is very important for a variety of activities both commercial and military. However, the high cost of IR cameras has limited their use to primarily scientific research and military applications. In order to detect IR radiation emitted from objects, the wavelength region between 3 to 5 $\mu \mathrm{m}$ and 8 to $14 \mu \mathrm{m}$ is used since it exhibits particularly low atmospheric absorption. Infrared radiation detectors ${ }^{1-4} \mathrm{can}^{\text {be }}$ classified broadly as either quantum (electro-optic) detectors ${ }^{5}$ or thermal detectors such as pyroelectric ${ }^{6}$, thermoelectric, resistive microbolometers ${ }^{7-10}$, and micromechanical thermal detectors ${ }^{11-18}$.

Among the various IR radiation detectors the class of quantum detectors have fast response times and high detectivities, $D^{\circ}$, but require the devices to be kept at a reduced temperature to minimize the effects of internal thermal noise that varies as $\exp \left(-\varepsilon_{g} / k T\right)$. For sensitive imaging in the mid- to far-infrared region, the photon detector must be cooled to liquid nitrogen temperatures $($ i.e. $\approx 77 \mathrm{~K}$ ) or lower, thus greatly increasing the cost, size and complexity of the detector. Thermal detectors have a very broadband response, since they are based upon thermal conversion of the absorbed energy. The thermoresistive microbolometer $r^{9,10,19,20}$ detector and a competing electric field enhanced pyroelectric ${ }^{6,21}$ array detector have demonstrated a noise equivalent temperature difference $(N E T D)$ of less than $50 \mathrm{mK}$; for a $320 \times 340$ array with $50 \mu \mathrm{m} \times 50 \mu \mathrm{m}$ pixel size an NETD of $<20 \mathrm{mK}$ was demonstrated ${ }^{19}$. Recently, a new type of thermal detector based on micromechanical structures was developed ${ }^{14,16-18,22}$ with a reported NEDT ranging from $5 \mathrm{~K}^{17}$ to $5 \mathrm{mK}^{16,22}$ and $D^{*} \sim 10^{8} \mathrm{~cm} \mathrm{~Hz}^{1 / 2} \mathrm{~W}^{-1}{ }^{14}$. However, these thermal detectors generally have slow response times $(>\mathrm{ms})$, low detectivities, and are limited by the fundamental temperature coefficient of resistance (for resistive bolometers), the pyroelectric coefficient (for pyroelectric detectors) and thermal bending 
sensitivity (for micromechanical systems). Hence fundamentally new detection techniques are required for the development of compact, light-weight, broadband, highly-sensitive, and low power consumption IR imaging sensors.

More recently we have developed a new approach for producing compact, light-weight, highly-sensitive micromechanical photon detectors that are based on micro-electro-mechanical systems (MEMS). This new type of semiconductor micromechanical detector operates as a quantum detector ${ }^{23-25}$. The operation relies on the interaction of photons directly with the semiconductor material or semiconductor/metal interface resulting in photo-induced stress. This stress causes the radius of curvature of the microstructure to change and provides an elegant way to detect photons.

Earlier work has shown that bending of microstructures can readily be determined by a number of means, including optical, capacitive, piezoresistive, and electron tunneling with extremely high sensitivity ${ }^{26,27}$. For example, the metal-coated microcantilevers that are commonly employed in AFM allow sub-Angstrom $\left(<10^{-10} \mathrm{~m}\right)$ sensitivity to be routinely obtained; Hansma ${ }^{27}$ and Binnig ${ }^{28}$ have demonstrated AFM sensitivities of $10^{-11} \mathrm{~N}$, corresponding to bending magnitudes of approximately $5 \times 10^{-11} \mathrm{~m}$. Recently, even smaller microcantilever deflections were measured with a resolution of $\sim 0.4 \times 10^{-12}$ $\mathrm{m}^{29,30}$. Standard AFM microcantilevers are typically 100-200 $\mu \mathrm{m}$ long, 0.3-3 $\mu \mathrm{m}$ thick and 10-30 $\mu \mathrm{m}$ wide, and can be fabricated from various dielectric or semiconducting materials. Microcantilevers made from GaAs were also fabricated with a thickness of merely $100 \mathrm{~nm}^{31}$. When even thinner microcantilevers were used, measurements of $10^{-18} \mathrm{~N}$ have been reported. In fact, when micromechanical structures are used as photon detectors and not as thermal detectors, they have faster response times and higher performance than that of micromechanical thermal detectors.

In our present studies we discuss both thermal and photonic microstructures. We investigated three systems: (i) $\mathrm{Si}$ microstructures, (ii) InSb micromechanical detectors and (iii) Si microstructures coated with a thin film of Pt. We found that for $\mathrm{Si}$ the photo-induced stress results in a contraction of the crystal lattice due to the presence of excess electron-hole-pairs while for InSb photo-induced stress causes the crystal lattice to expand. In the following sections we will present our results and discuss our findings.

\section{THERMALLY-INDUCED STRESS}

Bimaterial microstructures undergo bending due to differential surface stress in the microstructure. The change in the radius due to differential surface stress, $\Delta s$, can be written as ${ }^{14,32}$

$$
\frac{1}{R}=\frac{6\left(1+\left(t_{1} / t_{2}\right)^{2}\right)}{l\left(t_{1}+t_{2}\right)\left[3\left(1+t_{1} / t_{2}\right)^{2}+\left(1+t_{1} E_{1} / t_{2} E_{2}\right)\left(t_{1}^{2} / t_{2}^{2}+t_{2} E_{2} / t_{1} E_{1}\right)\right]} \frac{\Delta s}{E^{*}}
$$

where $t_{1}$ and $t_{2}$ are the thickness of the coating and microstructure substrate, $l$ is the microstructure length, $E_{1}$ and $E_{2}$ are the Young's moduli of the coating and microstructure, and $E^{*}$ is the effective Young's modulus of the coated microstructure. Note that $\vec{E}=E_{1} E_{2} /\left(E_{1}+E_{2}\right)$. Bending resulting from differential surface stress in such a microstructure can be detected with subnanometer sensitivities. The bending is related to the radius of curvature $z_{\max }=R^{2} /(2 R)$ which can be written as

$$
z_{\max }=\frac{3 l}{t_{1}+t_{2}}\left[\frac{1+\left(t_{1} / t_{2}\right)^{2}}{3\left(1+t_{1} / t_{2}\right)^{2}+\left(1+t_{1} E_{1} / t_{2} E_{2}\right)\left(t_{1}^{2} / t_{2}^{2}+t_{2} E_{2} / t_{1} E_{1}\right)}\right] \frac{\Delta s}{E^{*}} .
$$

Previous work has shown that silicon nitride microcantilevers with a thin gold film on one side undergo measurable bending due to temperature changes as small as $10^{-6} \mathrm{~K}$. This bending is due to the differential stress created by dissimilar thermal expansion of the microcantilever substrate and the gold coating (or the bimetallic effect). The differential stress due to thermal expansion of these materials can be approximated as ${ }^{14}$

$$
\Delta s \approx\left(E_{1} \alpha_{1}-E_{2} \alpha_{2}\right) l \Delta T
$$


where $\Delta T$ is the temperature change of the microstructure and $\alpha_{1}$ and $\alpha_{2}$ are the coefficients of thermal expansion for the materials composing the bimetallic strip. By measuring the bending distance $z_{\max }$, the change in temperature can be determined by

$$
z_{\max }=\frac{3 l^{2}}{t_{1}+t_{2}}\left[\frac{1+\left(t_{1} / t_{2}\right)^{2}}{3\left(1+t_{1} / t_{2}\right)^{2}+\left(1+t_{1} E_{1} / t_{2} E_{2}\right)\left(t_{1}^{2} / t_{2}^{2}+t_{2} E_{2} / t_{1} E_{1}\right)}\right] \frac{E_{1} \alpha_{1}-E_{2} \alpha_{2}}{E^{*}} \Delta T
$$

This assumes the ideal case where the microstructure and its base are at the same temperature. This also assumes that all incident radiation is absorbed by the microstructure and the base, resulting in a uniform temperature change. The fundamental limits to the performance of these micromechanical structures are established by the properties of the microstructures themselves and also depend on the readout means. When an optical readout scheme is used to measure $z_{\max }$, in certain cases the influence of the optical readout can be negligible. The fundamental limits i.e., background fluctuation noise limit and temperature fluctuation noise limit depend on the inherent properties of the microstructures. An ideal, noiseless readout would amplify and display the signal and noise inherent to the microstructures without adding any excess noise. It turns out that the optical readout used in this work is very close to this case.

\section{ELECTRONICALLY-INDUCED STRESS}

Microstructures exposed to photons (see Figure 1) undergo bending due to the differential surface stress ${ }^{33}\left(\Delta s=s_{1}-s_{2}\right)$ created by differential expansions. When the structure is bimaterial the bending is due to the differentiated response of the two materials. Earlier work has shown that the absorption of photons by a solid results in temperature changes and thermal expansion which in turn gives rise to acoustic waves at frequencies corresponding to the amplitude modulation of the incident photon beam ${ }^{34,35}$. In a semiconductor the generation of "free" charge carriers (electrons and holes) via photon irradiation results in the development of a local mechanical strain ${ }^{36,37}$. This additional strain adds to other strains resulting from temperature changes in the semiconductor material. When the photon flux is high enough to cause the semiconductor to heat, the subsequent expansion (or contraction) can be detected as acoustic waves with conventional photoacoustic techniques ${ }^{35,38}$.

In a semiconductor structure of thickness $t$ and
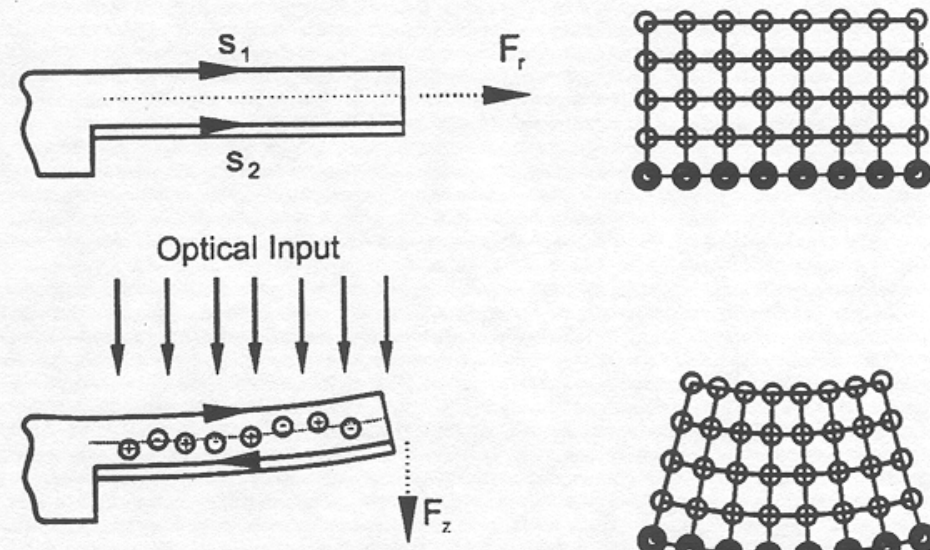

Figure 1. Schematic diagram showing the bending process of a semiconductor microstructure exposed to photons. Surface stresses $s_{1}$ and $s_{2}$ are balanced at equilibrium. Also depicted is the accompanied contraction of a semiconductor lattice following the generation of electron hole pairs.

energy bandgap $\varepsilon_{g}$, the change in total surface stress due to photogenerated change in the charge carrier density, $\Delta n$ will be given by $23,36,37,39$

$$
\Delta s=\Delta s_{p i}=\left(\frac{1}{3} \frac{d \varepsilon_{g}}{d P} \Delta n\right) E
$$

where, $d \varepsilon_{g} / d P$ is the pressure dependence of the energy bandgap, $\alpha$ is the coefficient of thermal expansion, and $E$ is the Young's modulus. When $d \varepsilon_{g} J d P$ is negative the photo-induced stress is of opposite sign than that of the thermal stress and will tend to make the semiconductor crystal to contract. The deflection sensitivity can be enhanced by coating the microstructure with a 
layer of another material. For a rectangular bimaterial bar (Figure 1) of length $l$, width $w$, and total thickness $t$, the reciprocal of the radius of curvature, $R$, is given by

$$
\frac{1}{R}=\frac{2\left(1+\left(t_{1} / t_{2}\right)^{2}\right)}{\left(t_{1}+t_{2}\right)\left[3\left(1+t_{1} / t_{2}\right)^{2}+\left(1+t_{1} E_{1} / t_{2} E_{2}\right)\left(t_{1}^{2} / t_{2}^{2}+t_{2} E_{2} / t_{1} E_{1}\right)\right]}\left(\frac{d \varepsilon_{g}}{d P} \Delta n\right) \frac{E}{E^{\bullet}}
$$

where $t_{1}$ and $t_{2}$ are the thickness of the bimaterial layer and microstructure substrate, $E_{1}$ and $E_{2}$ are the Young's moduli of the bimaterial layer and microstructure, and $E^{*}$ is the effective Young's modulus of the coated microstructure and is given by $E^{*}=$ $E_{1} E_{2} /\left(E_{1}+E_{2}\right)$. Materials with large differences in their $E$ will offer better deflection sensitivity. However, the larger the difference between the Young's modulus the more difficult it becomes to deposit a bimaterial layer and not produce "curled" microstructures ${ }^{16,30}$. The deposition of metal layers on thin microcantilevers to produce unstressed structures with no bending is difficult and requires extremely high thermal stability. Bimaterial microcantilevers with no noticeable bending have been produced when care was taken to avoid any temperature rises during the bimaterial deposition process ${ }^{30}$. In those studies the investigators broke down the complete deposition process into 20 steps in order to avoid the temperature of the microcantilever rising during the deposition.

The reciprocal of the radius of curvature is approximately equal to $d^{2} z / d y^{2}{ }^{40}$. Then, using Eqn (6) the maximum displacement $z_{\max }$ of the microstructure is given by

$$
z_{\max }=\frac{l^{2}}{t_{1}+t_{2}}\left(\frac{1+\left(t_{1} / t_{2}\right)^{2}}{3\left(1+t_{1} / t_{2}\right)^{2}+\left(1+t_{1} E_{1} / t_{2} E_{2}\right)\left(t_{1}^{2} / t_{2}^{2}+t_{2} E_{2} / t_{1} E_{1}\right)}\right) \frac{E_{1}}{E^{*}} \frac{d \varepsilon_{g}}{d P} \Delta n
$$

The overall change in $z_{\max }$ will depend on several physical and mechanical properties of the semiconductor. In fact, for the same input power a Si microstructure exhibits a photo-induced bending that is about four times larger than that due to thermal induced stress ${ }^{23}$.

Assuming that an incident radiant power, $\Phi_{e}$, in a semiconductor microstructure generates number density of excess charge carriers, $\Delta n$, we get

$$
\Delta n=\eta \frac{\lambda}{h c} \frac{\tau_{L}}{l w\left(t_{1}+t_{2}\right)} \Phi_{a}^{a b s}
$$

where $\eta$ is the quantum efficiency, $h\left(=6.625 \times 10^{-34} \mathrm{~J} \mathrm{~s}\right)$ is Planck's constant, $c\left(=3 \times 10^{8} \mathrm{~m} \mathrm{~s}^{-1}\right)$ is the speed of light, and $\tau_{L}$ is the lifetime of the carriers in the semiconductor. Then the maximum displacement $z_{\max }$ can be rewritten as

$$
z_{\max }=\frac{l}{w\left(t_{1}+t_{2}\right)^{2}}\left(\frac{1+\left(t_{1} / t_{2}\right)^{2}}{3\left(1+t_{1} / t_{2}\right)^{2}+\left(1+t_{1} E_{1} / t_{2} E_{2}\right)\left(t_{1}^{2} / t_{2}^{2}+t_{2} E_{2} / t_{1} E_{1}\right)}\right) \frac{E_{1}}{E^{*}} \eta \frac{\lambda}{h c} \frac{d \varepsilon_{g}}{d P} \tau_{L} \Phi_{e}^{a b s} .
$$

We can define the deflection responsivity $\Re=z / \Phi_{e}$, viz.,

$$
\Re=\frac{l}{w\left(t_{1}+t_{2}\right)^{2}}\left(\frac{1+\left(t_{1} / t_{2}\right)^{2}}{3\left(1+t_{1} / t_{2}\right)^{2}+\left(1+t_{1} E_{1} / t_{2} E_{2}\right)\left(t_{1}^{2} / t_{2}^{2}+t_{2} E_{2} / t_{1} E_{1}\right)}\right) \frac{E_{1}}{E^{*}} \eta \frac{\lambda}{h c} \frac{d \varepsilon_{g}}{d P} \tau_{L} .
$$


Since the charge carriers can be generated in a very short time the photo-induced stress can manifest itself much faster than thermal stress.

In Figure 2 we calculated the photo-induced responsivity as a function of photon wavelength for a number of different semiconductor materials using Eqn (10) and the material properties found in Table I. We assumed the microstructures had length $l=100 \mu \mathrm{m}$, width $w=20 \mu \mathrm{m}$, and total thickness $t=0.5 \mu \mathrm{m}$. The bending due to photo-generated charges increases linearly with increasing wavelength up to the cutoff $\lambda_{c}$, of several different semiconductor materials. Semiconductor microstructures with longer wavelengths are required for detection of IR radiation emitted from objects at room temperature. It is interesting to note that InSb has both longer cutoff wavelength and higher responsivity.

Table I. Properties of semiconductor materials that can be used as micromechanical photon detectors.

\begin{tabular}{|c|c|c|c|c|}
\hline $\begin{array}{l}\text { Semiconductor } \\
\text { Material }\end{array}$ & $\begin{array}{c}\varepsilon_{\mathrm{g}} \\
(\mathrm{eV})\end{array}$ & $\begin{array}{c}d \varepsilon_{\mathrm{g}} / d P \\
\left(10^{-24} \mathrm{~cm}^{3}\right)\end{array}$ & $\begin{array}{c}E \\
(\mathrm{GPa})\end{array}$ & $\begin{array}{c}G \\
\left(\mathrm{~W} \mathrm{~m}^{-1} \mathrm{~K}^{-1}\right)\end{array}$ \\
\hline GaAs & $1.35^{\mathrm{a}}$ & $-13.67^{b}$ & 85.5 & 55 \\
\hline $\mathrm{Si}$ & $1.12^{\mathrm{a}}$ & $-3.14^{c}$ & 130.91 & 163 \\
\hline $\mathrm{Ge}$ & $0.67^{\mathrm{a}}$ & $11.52^{c}$ & 102.66 & 59 \\
\hline InSb & $0.16^{\mathrm{a}}$ & $23.61^{c}$ & 42.79 & 36 \\
\hline \multicolumn{5}{|c|}{$\begin{array}{l}\text { a From reference [4]. } \\
\text { b From reference [41]. } \\
{ }^{\mathrm{c}} \text { From reference [42]. }\end{array}$} \\
\hline
\end{tabular}

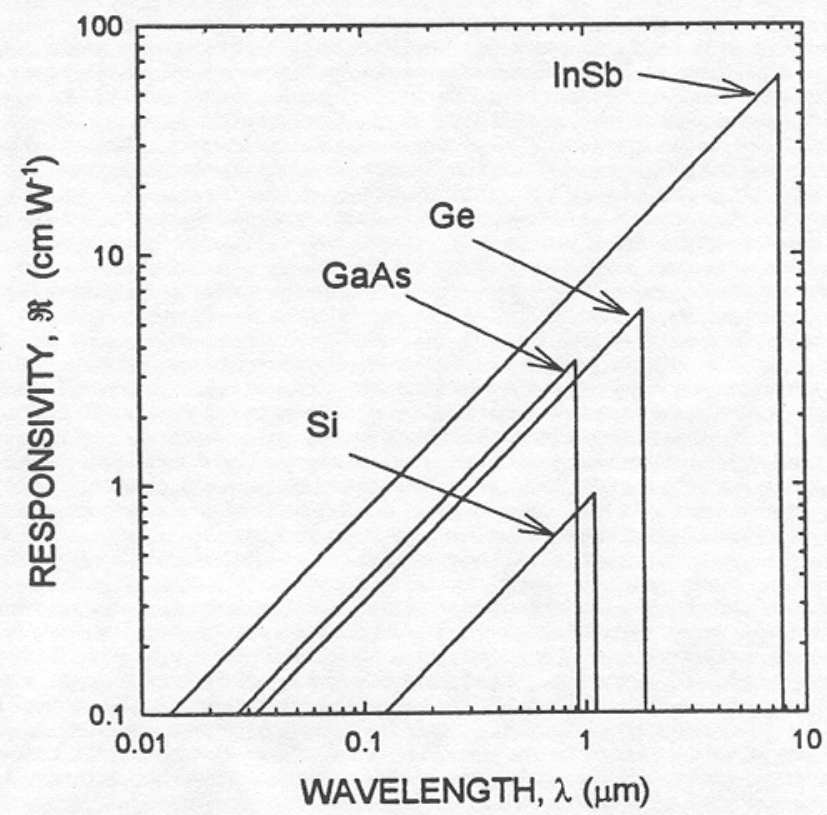

Figure 2. Calculated responsivity, $\Re$, for different semiconductor microstructures as a function of photon wavelength, for different materials. 


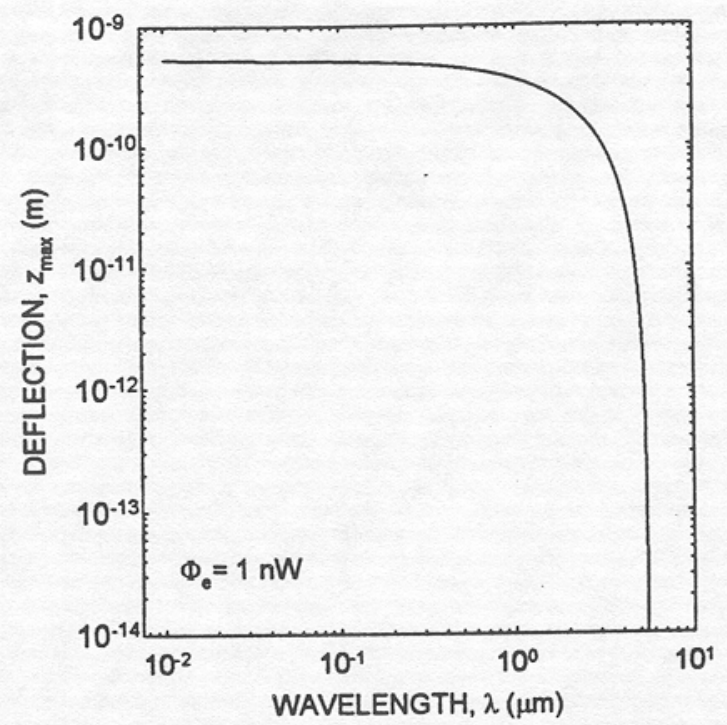

Figure 3. Calculated deflection of a Pt-Si quantum detector due to internal photoemission stress as a function of photon wavelength for an absorbed power of $1 \mathrm{nW}$.

\section{PHOTO-INDUCED ELECTRONIC STRESS IN METAL-SEMICONDUCTOR}

Photo-induced stress can be manifested in semiconductor microstructures with a metal/semiconductor interface which forms a Schottky barrier. When such a device is exposed to photons (with energies above the Schottky barrier), the excess charge carriers generated induce an electronic stress, which causes changes in the radius of curvature of the microstructure and the microstructure bends. The extent of bending is directly proportional to the radiation intensity.

Again for a rectangular bar (Figure 1) of length $l$, width $w$, and total thickness $t$, the maximum displacement $z_{\max }$ is given by Eqn (9). However, the overall change in $z_{\max }$ will depend on several physical and mechanical properties of the metal/semiconductor system. The quantum efficiency for a Schottky barrier can be described as ${ }^{4}$

$$
\eta=C_{0} \frac{(h c / \lambda-\Psi)^{2}}{h c / \lambda}=C_{0} \frac{h c}{\lambda}\left(1-\frac{\Psi \lambda}{h c}\right)^{2}
$$

where $C_{0}$ is in units of inverse energy and depends on the quantum yield and $\Psi$ is the Schottky barrier height. Then the maximum displacement $z_{\max }$ can be rewritten as

$$
z_{\max }=C_{0} \frac{l}{w\left(t_{1}+t_{2}\right)^{2}}\left(\frac{1+\left(t_{1} / t_{2}\right)^{2}}{3\left(1+t_{1} / t_{2}\right)^{2}+\left(1+t_{1} E_{1} / t_{2} E_{2}\right)\left(t_{1}^{2} / t_{2}^{2}+t_{2} E_{2} / t_{1} E_{1}\right)}\right) \frac{E_{1}}{E^{*}}\left(1-\frac{\lambda}{\lambda_{c}}\right)^{2} \frac{d \varepsilon_{g}}{d P} \tau_{L} \Phi_{G}^{a b s}
$$

where $\lambda_{\mathrm{c}}(=h c / \Psi)$ is the cutoff wavelength for photoemission form the Schottky interface. Since the charge carriers can be generated in a very short time the photo-induced stress can manifest itself much faster than thermal stress. We can then define a deflection responsivity $\Re=z / \Phi_{e}$, viz., 


$$
\Re=C_{0} \frac{l}{w\left(t_{1}+t_{2}\right)^{2}}\left(\frac{1+\left(t_{1} / t_{2}\right)^{2}}{3\left(1+t_{1} / t_{2}\right)^{2}+\left(1+t_{1} E_{1} / t_{2} E_{2}\right)\left(t_{1}^{2} / t_{2}^{2}+t_{2} E_{2} / t_{1} E_{1}\right)}\right) \frac{E_{1}}{E^{*}}\left(1-\frac{\lambda}{\lambda_{c}}\right)^{2} \frac{d \varepsilon_{g}}{d P} \tau_{L} .
$$

From Eqn (12) we can calculate the deflection responsivity for a Pt-Si system (which can form Platinum Silicide) using values found in the literature ${ }^{41}$ for $\operatorname{Si}\left(d \varepsilon_{g} / d P=-3.14 \times 10^{-24} \mathrm{~cm}^{3}\right.$, and $\left.E_{1}=1.69 \times 10^{12} \mathrm{dyn} / \mathrm{cm}^{2}\right)$ and for Pt $\left(E_{2}=1.91 \times 10^{12} \mathrm{dyn} / \mathrm{cm}^{2}\right)$, the photo-induced deflection of a Pt-Si microstructure photon detector as a function of photon wavelength; the absorbed power was assumed to be $1 \mathrm{nW}$. The Pt-Si microstructure photon detector was given a length $l=100 \mu \mathrm{m}$, width $w=20 \mu \mathrm{m}$, total thickness $t=500 \mathrm{~nm}$ and a $30 \mathrm{~nm}$ Pt coating. The overall bending due to internal photoemission is plotted in Figure 3 and can be seen to decrease with increasing wavelength up to the cutoff wavelength of $\mathrm{PtSi}\left(\lambda_{c}=5.5 \mu \mathrm{m}\right)$.

\section{EXPERIMENTAL}

Although bending of microstructures can readily be determined by a number of means (optical, capacitive, electron tunneling, and piezoresistive methods), in this work we employed an optical readout technique. It turns out that the optical readout technique adds negligible noise in the detector system. The approach used was adapted from standard atomic force microscopy imaging systems, and is shown schematically in Figure 4. Microstructures were mounted in a chip holder which, in turn, was mounted on a three-axis translation stage to facilitate fine adjustment of the microstructure relative to the rest of the experimental apparatus. A mechanical chopper was used to modulate the incoming photon radiation. This configuration provided a flexible, easily controlled test system for quantifying microstructure response to optical energy. All measurements were conducted at ambient temperature and atmospheric conditions.

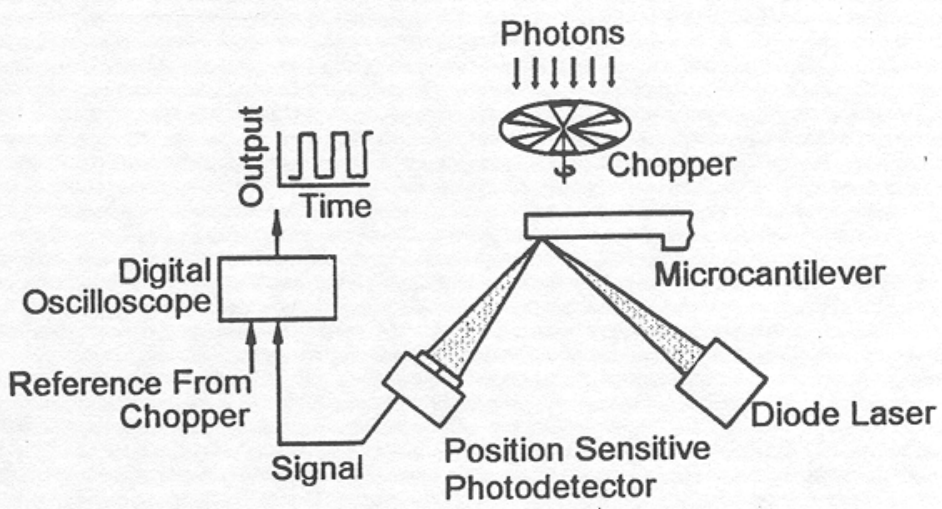

Figure 4. Schematic diagram of the experimental setup used in the present studies. A read laser beam is focused onto the tip of the microstructure and the incident radiation is modulated using a chopper or by pulsing the emitting source.

A second laser was used in a probe configuration to monitor bending. A laser beam was focused onto the tip of the microstructure using and in order to minimize heating of the tip by the probe laser, optical power was reduced by placing a neutral density filter. A quad-element $(A, B, C, D)$ photodiode detector was used to collect the reflected probe beam. The current output $\left(i_{\mathrm{A}, \mathrm{B}, \mathrm{C}, \mathrm{D}}\right)$ of the photodiode depends linearly on the bending of the microstructure. A high narrow bandpass optical filter is placed in front of the photodiode allowing the laser beam to be detected while preventing other wavelengths from reaching the photodiode. The amplified differential current signal from the quad cell photodiode, $i_{\mathrm{A}, \mathrm{B}, \mathrm{C}, \mathrm{D}}\left[=\left(i_{\mathrm{A}}+i_{\mathrm{B}}\right)-\left(i_{\mathrm{C}}+i_{\mathrm{D}}\right) /\left(i_{\mathrm{A}}+i_{\mathrm{B}}+\right.\right.$ $\left.\left.i_{\mathrm{C}}+i_{\mathrm{D}}\right)\right]$, is monitored and recorded using a digital oscilloscope (Tektronix TDS 780) or sent to a lock-in amplifier (Stanford Research Systems SR850) for signal extraction and averaging.

During our studies we also coated $\mathrm{Si}$ microstructures with a thin layer of $\mathrm{Al}(30 \mathrm{~nm})$. We used an argon ion beam and an $\mathrm{Al}$ target to sputter a thin coating on one surface of microstructures; we used Al coated microstructures to study the effect of thermal stress using a diode laser with $1300 \mathrm{~nm}$ photons. We found that even at those small thicknesses, $\mathrm{Al}$ causes the microstructures to "curl". We heated the coated microstructures to about $500^{\circ} \mathrm{C}$ for a period of four to six hours to reduce residual stresses. This procedure seemed to result in microstructures with almost no residual bending. However, the resonance frequency of the 
coated microstructures was lower than the uncoated and it is rather difficult to determine how much of the shift was due to mass loading and how much due to residual stresses.

Our InSb structures were microfabricated using single point diamond and ion beam milling processes. A focused ion beam (FIB) system was used to "write" the desired patterns and shape the detector element. Al (50 nm) was slowly deposited on one side of the InSb microstructure to make it bimaterial. We fabricated platinum silicide microstructures by coating $\mathrm{Si}$ microstructures with a thin layer of $\mathrm{Pt}(30 \mathrm{~nm})$. The coated devices were subsequently placed in vacuum chamber and heated to $450^{\circ} \mathrm{C}$ for a period of three to four hours in order to produce platinum silicide at the interface of $\mathrm{Si}$ and $\mathrm{Pt}$. An additional benefit of this process was the fact that the resulting microstructures had little or none residual stress present as a result of the deposition process.

\section{RESULTS}

The microstructures were exposed to photons from a diode laser with wavelength $\lambda=780 \mathrm{~nm}, 1300 \mathrm{~nm}$ and $1550 \mathrm{~nm}$, and using a mechanical chopper, the incoming photon radiation was modulated at different frequencies. We measured the bending of microstructures as a function of time and in Figure 5 we plotted that temporal response of a Si microstructure when the absorbed optical power was $3.9 \mathrm{nW}$; the absorbed power was calculated using $\Phi_{e}^{a b s}=\alpha_{\mathrm{abs}} \Phi_{e}^{\text {inc }} \mathrm{A}_{\mathrm{Al}} / \mathrm{A}_{\text {spot }}$ where $\alpha_{\mathrm{abs}}=0.95$ is the absorptivity of $\mathrm{Si}$ at $780 \mathrm{~nm}, \mathrm{~A}_{d}$ is the microstructure area and $\mathrm{A}_{\text {spot }}\left(=1.53 \mathrm{~mm}^{2}\right)$ is the area of the focused laser beam at the plane of the microstructure. The observed bending is attributed to the fact that the irradiated (top) side of the microstructure coatedct sooner that the bottom side. As can be seen from Figure 5 the Si microstructure responds rapidly to incoming photons that generate charge carriers which, in turn, cause a measurable mechanical bending. In these experiments, photons continued to impinge on the detector surface for about $5 \times 10^{-4} \mathrm{~s}$, while the bending reached its maximum value within $1 \times 10^{-4} \mathrm{~s}$. The time

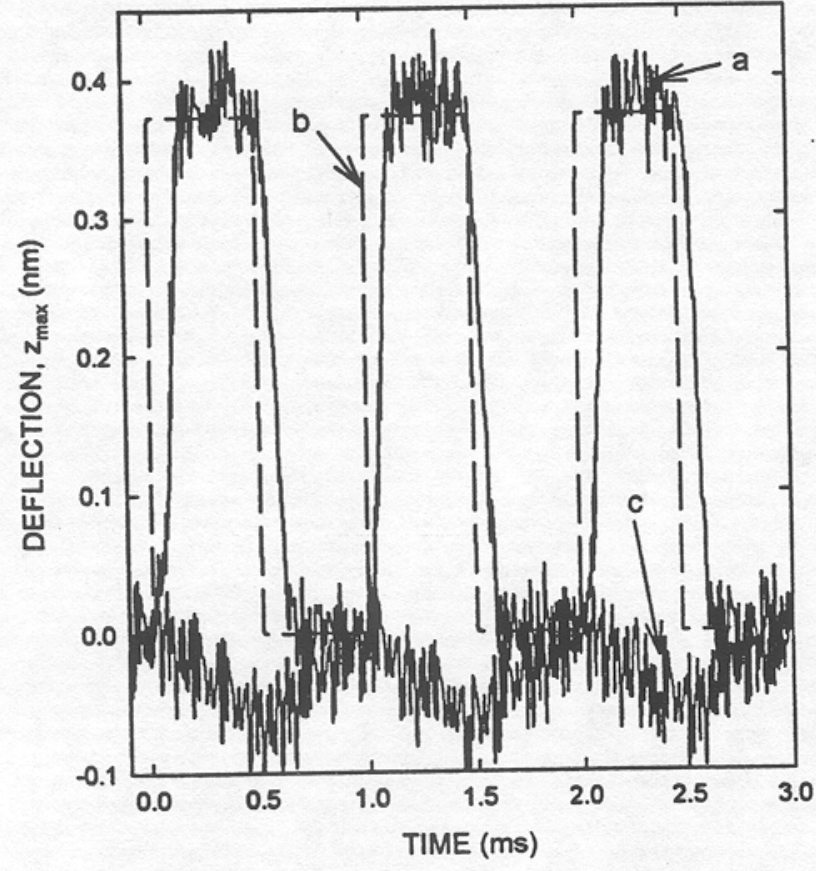

Figure 5. Deflection of a Si microstructure [curve (a)] due to photo-generated charge carrier when exposed to photons with $\lambda=780 \mathrm{~nm}$ and an absorbed power of $3.9 \mathrm{nW}$. The dashed curve (b) represents the signal from the modulator and shows the amount of time the detector was exposed to photons. Curve (c) corresponds to deflection of the microstructure due to absorption of $2 \mathrm{nW}$ power of photons with $\lambda=1300 \mathrm{~nm}$ and is purely due to thermal effects.

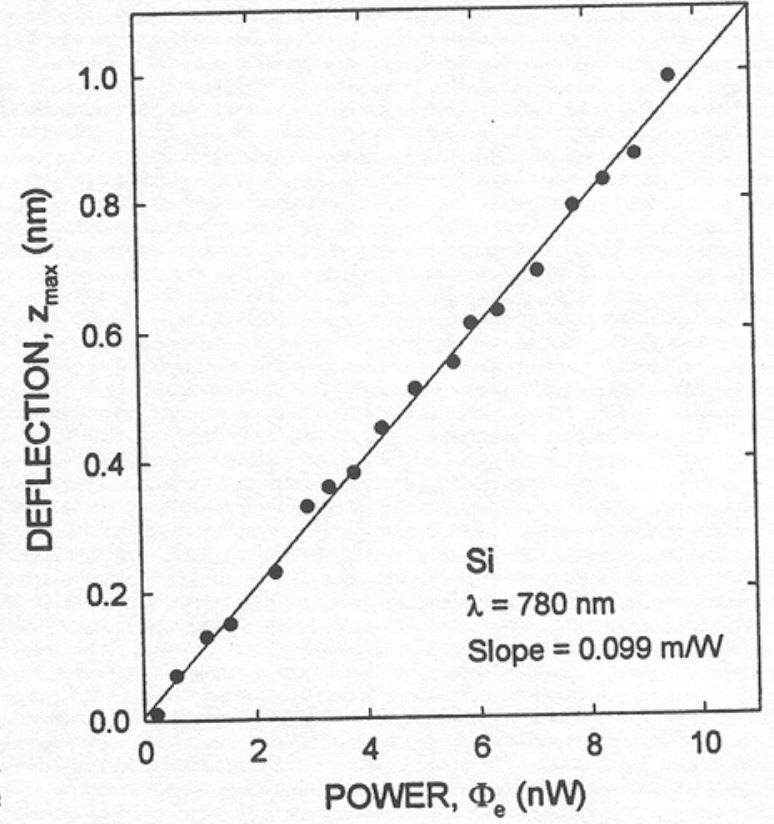

Figure 6. Deflection of a Si microstructure as a function of absorbed power due to photo-generated charge carrier when exposed to photons with $\lambda=780 \mathrm{~nm}$. 
that the microstructure reached its maximum bending corresponds nicely to the lifetime of photo-generated charge carriers in Si. For a similar structure, thermal effects have been found to play a role in a slower time scale and have a time constant $>10^{-3} \mathrm{~s}^{1214}$.

In order to determine the effect of the thermal stress under our experimental conditions, we illuminated the Si microstructure using photons with $\lambda=1300 \mathrm{~nm}$. Since $\mathrm{Si}$ is transparent to these photons, we deposited a thin aluminum coating of $30 \mathrm{~nm}$ along one side of the Si microstructure using the procedure described in above. The absorbed power was estimated using $\Phi_{e}^{\text {abs }}=$ $\alpha_{\text {abs }} \Phi_{e}^{\text {inc }} \mathrm{A}_{\mathrm{Al}} / \mathrm{A}_{\text {spot }}$ where $\alpha_{\mathrm{abs}}=0.01$ is the absorbivity of $\mathrm{Al}$ and $\mathrm{A}_{\mathrm{Al}}\left(=2 \times 10^{-5} \mathrm{~cm}^{2}\right)$ is the area of the $\mathrm{Al}$ coating. We modulated the infrared radiation at a frequency of $1000 \mathrm{~Hz}$ and in Figure 5 curve (c) we plotted the measured the microstructure bending as a function of time for an absorbed power of $2 \mathrm{nW}$. The change in the direction of microstructure bending compared to curve (a), is due to the fact that the irradiated (top) side of the microstructure expands sooner that the bottom side. From curve (c) in Figure 5 it can be seen that for $780 \mathrm{~nm}$ photons and $1000 \mathrm{~Hz}$ modulation frequency, the observed thermally induced bending of the microstructure is smaller (over 2 times after correcting for the absorbed power) compared to the photo-induced bending and the response time is longer (>ms). The response of the microstructure [curve (a) in Figure 5] is faster than deflections due to thermal effects [curve (c) in Figure 5].

Since $d \varepsilon_{g} / d P$ is negative for $\mathrm{Si}^{41}$, it should be straightforward to distinguish the photo-induced stress from the thermal stress in $\mathrm{Si}$ by observing the direction of bending indicated by the phase shift of the signal waveform with respect to the reference signal. When photons with energies above the bandgap are used, the Si microstructure contracts and deflects in one direction [curve (a) in Figure 5]. However, when photons with energies below the bandgap are used, the Si beam expands and deflects in the opposite direction [curve (c) in Figure 5]. We also measured the microstructure bending due to photo-induced stress as a function of absorbed power. In Figure 6 we plotted the measured bending of a Si microstructure as a function of absorbed power using a diode laser with $\lambda=780 \mathrm{~nm}$. The microstructure deflection was primarily due to photo-induced stress and was found to increase linearly with increasing power with a deflection sensitivity of $0.099 \mathrm{~m} / \mathrm{W}$.

We also investigated the response of InSb microstructures to photons and measured the microstructure bending due to electronic stress as a function of absorbed power. We calculated the absorbed power using $\Phi_{e}^{a b s}=\alpha_{\mathrm{abs}} \Phi_{e}^{i n c} A_{\mathrm{Al}} / \mathrm{A}_{\text {spot }}$ and in Figure 7 we

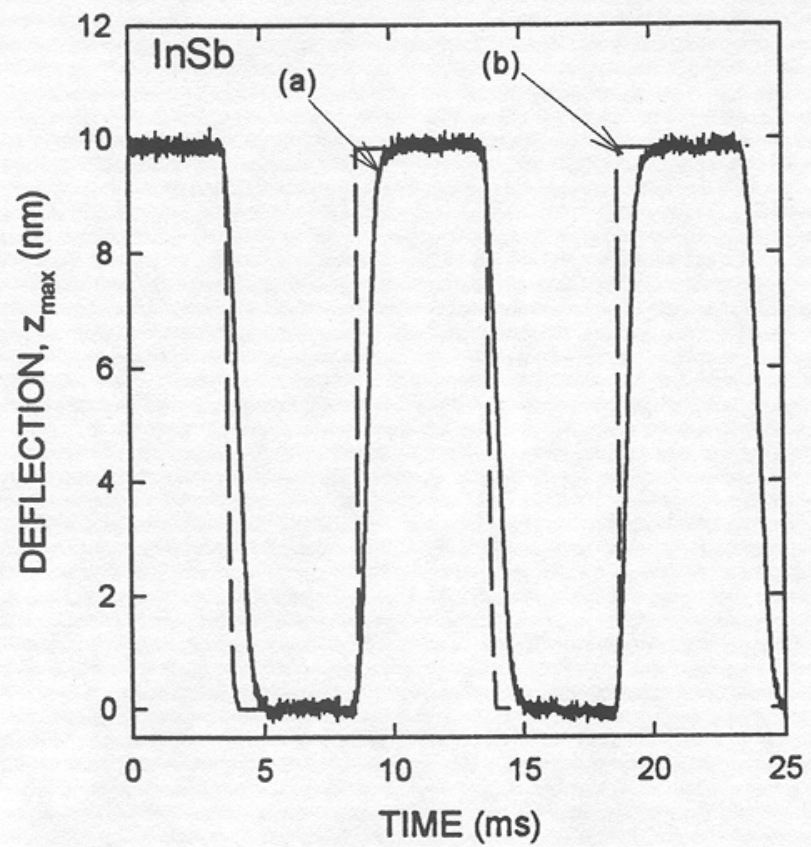

Figure 7. Deflection of a InSb microstructure [curve (a)] due to photo-generated stress. The dashed curve (b) represents the signal from the modulator and shows the amount of time the detector was exposed to photons.

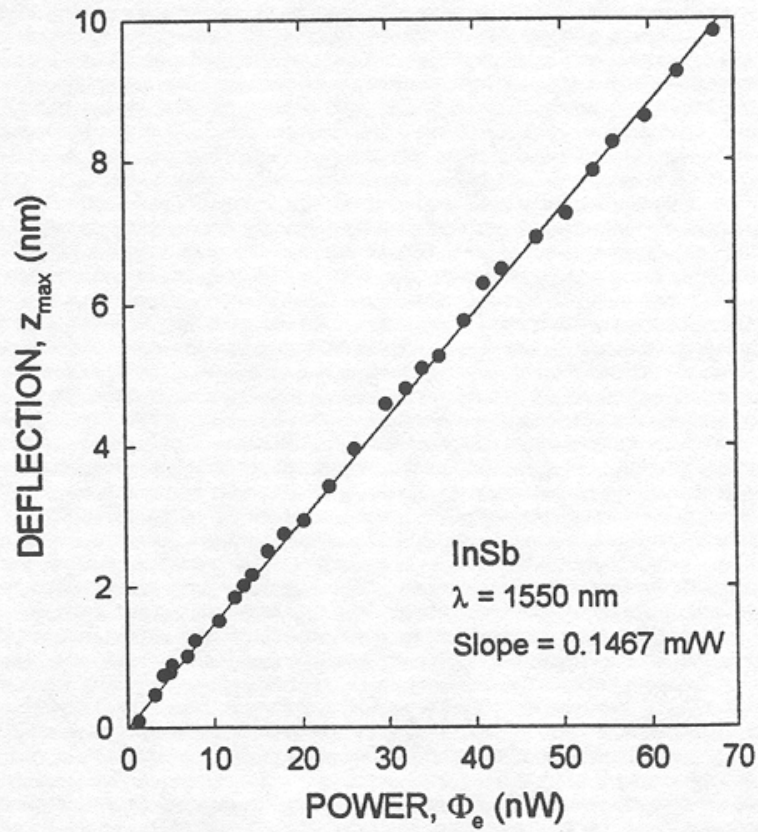

Figure 8. Deflection of an InSb microstructure as a function of absorbed power due to photo-induced stress when exposed to photons with $\lambda=1500 \mathrm{~nm}$. 


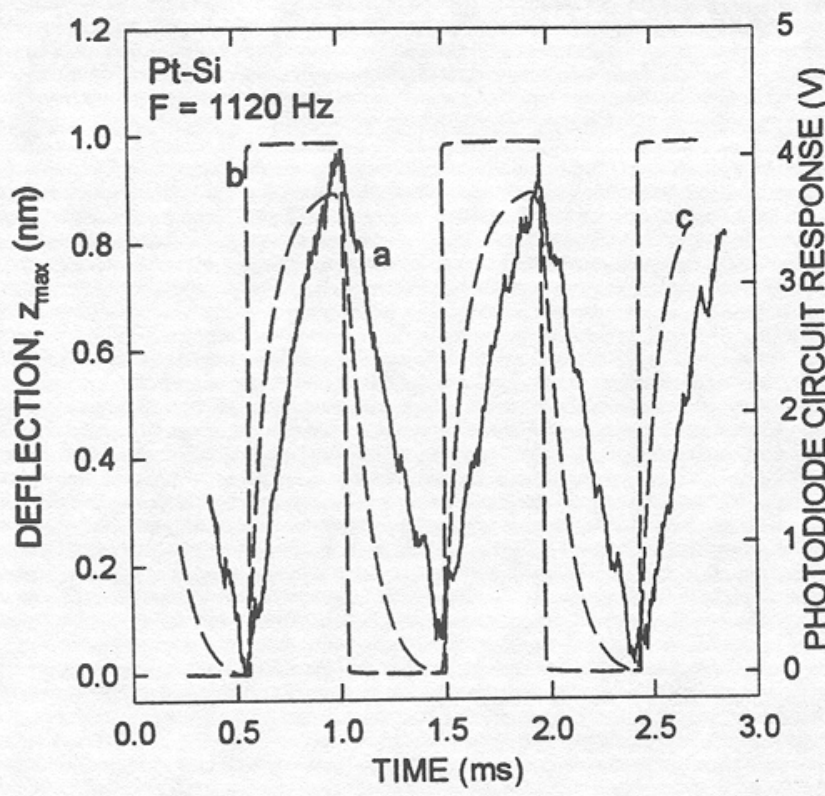

Figure 9. Deflection of a Pt-Si microstructure [curve (a)] due to internal photoemission at the Pt-Si interface when exposed to photons with $\lambda=1550 \mathrm{~nm}$ and an absorbed power of $20 \mathrm{nW}$. The dashed curves (b) and (c) are associated with the left vertical axis. The dashed curve (b) represents the signal from the modulator and shows the amount of time the Pt-Si microstructure was exposed to photons. The dashed curve (c) is the signal from the quad cell photodiode and shows the time response of our optical position measuring circuit.

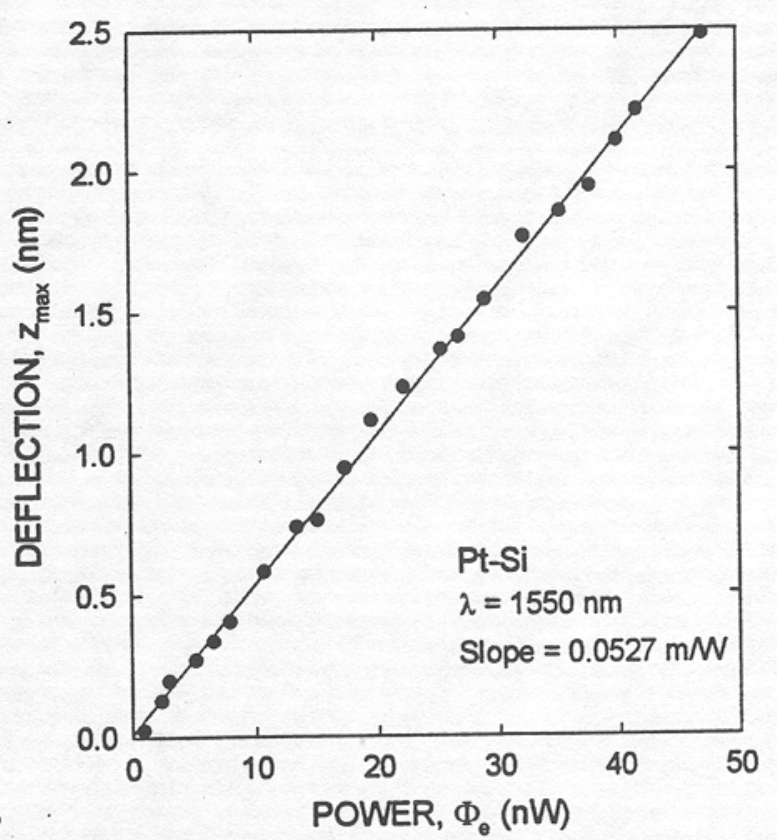

Figure 10. Deflection of a Pt-Si microstructure as a function of absorbed power due to internal photoemission when exposed to photons with $\lambda=1500 \mathrm{~nm}$.

plotted the deflection of an InSb microstructure as a function of time due to photo-induced stress when exposed. In Figure 8 we plotted the measured bending of an InSb microstructure as a function of absorbed power using a diode laser source with wavelength $\lambda=1550 \mathrm{~nm}$. The deflection of the InSb microstructure was primarily due to electronic stress caused by excess charge carriers in the conduction band and was found to increase linearly with increasing power with a deflection sensitivity of $0.1467 \mathrm{~m} / \mathrm{W}$ (see Figure 8). In this work the smallest positional changes we measured were a fraction of a nanometer.

Pt-Si microstructures were exposed to photons from a diode laser with wavelength $\lambda=1550 \mathrm{~nm}$ and using a mechanical chopper, the incoming photon radiation was modulated at a frequency of $1120 \mathrm{~Hz}$. Since Si is transparent to photon wavelengths above $1100 \mathrm{~nm}, 1550 \mathrm{~nm}$ photons can reach the interface of $\mathrm{Pt}$ and $\mathrm{Si}$ and generate photoelectrons from platinum silicide. These electrons can drift into $\mathrm{Si}$ and cause an electronic stress. We measured the deflection of Pt-Si microstructure as a function of time and in Figure 9 plotted the temporal response when the absorbed optical power was $20 \mathrm{nW}$. The absorbed power was calculated using $\Phi_{e}^{a b s}=\alpha_{\text {abs }} \Phi_{e}^{\text {inc }} \times \mathrm{A}_{d} / \mathrm{A}_{\text {spot, }}$, where $\alpha_{\text {abs }}$ (roughly 0.01 ) is the photon absorptivity of Pt at $1550 \mathrm{~nm}, \mathrm{~A}_{d}$ is the microstructure area and $A_{\text {spot }}\left(=1.75 \mathrm{~mm}^{2}\right)$ is the area of the focused laser beam at the plane of the microstructure. As can be seen from Figure 9, the Pt-Si microstructure responds rapidly to incoming photons that generate photoelectron from the Pt-Si surface which, in turn, cause a measurable mechanical bending. For similar structures, thermal effects have been found to play a role in slower time scales with a time constants $>10^{-3} \mathrm{~s}^{11,12,14}$. Since the pressure dependence of the bandgap is negative for $\mathrm{Si}^{41}$, excess electrons present in the $\mathrm{Si}$ will cause $\mathrm{Si}$ to contract while the Pt layer will either expand or remain unaffected. It is this bimaterial effect that makes the micromechanical structure exhibit increased bending when exposed to infrared photons. We should point out that the temporal response of the Pt-Si microstructure shown in Figure 9 (solid curve a) is limited by the time constant of optical read-out electronics. This observation is supported by the response of the read-out quad cell photodiode shown in Figure 9 (dashed curve c). It can be seen that the photodiode response is dramatically influenced by the time constant of the read-out circuit. 
We also exposed our Pt-Si microstructure to varying input radiant power and measured the microstructure bending due to electronic stress as a function of absorbed power. Again, we calculated the absorbed power using $\Phi_{e}^{\text {abs }}=\alpha_{\text {abs }} \Phi_{e}{ }^{\text {ine }} \times A_{\mathrm{d}} / A_{\text {spot }}$ and in Figure 10 we plotted the measured bending of a Pt-Si microstructure as a function of absorbed power using a diode laser source with wavelength $\lambda=1550 \mathrm{~nm}$. The deflection of the Pt-Si microstructure was primarily due to electronic stress caused by internal photoemission and was found to increase linearly with increasing power with a deflection sensitivity of $0.0527 \mathrm{~m} / \mathrm{W}$. In our studies the smallest positional changes we measured were a fraction of a nanometer. However, much smaller values are possible $\left(10^{-12} \mathrm{~m}\right)$ corresponding to a minimum detectable power of $10^{-11} \mathrm{~W}$ which, in turn, translates to a detectivity of $10^{9} \mathrm{~cm} \mathrm{~Hz}^{1 / 2} \mathrm{~W}^{-1}$ at $30 \mathrm{~Hz}$. This value is one order of magnitude higher than the $D^{*}$ reported for microstructure thermal detectors $^{14}$.

Our results demonstrate that exposing Pt-Si microstructures to photons with energies above the PtSi Schottky barrier produces a photo-induced mechanical stress, which dominates over thermally-induced stresses. However, when the photon energy is below the PtSi Schottky barrier, thermal effects dominate. We found that the deflection of Pt-Si microstructures depend linearly on photoemissively-induced stress which, in turn, depends linearly on the input optical power and is manifested with fast response times. Furthermore, this mechanism does not rely on changes in the temperature of the microstructure and therefore thermal isolation (that is crucial to the operation of thermal detectors) has minimal influence.

Utilizing photon detection mechanisms that rely on the measurement of photo-induced stress in micromechanical structures, it may be possible to construct micromechanical photon detector arrays using microfabrication processes. Unlike thermal detectors that respond slowly (response times $\sim \mathrm{ms}$ ) to impinging photons via temperature changes, a detector based on the photo-induced stress will respond both more sensitively and rapidly to incoming photons, with fast response times $<$ ms. Usually, the sensitivity of micromechanical photon increases as the thickness to length ratio of the microstructure decreases. However, in devices with smaller thickness the photon absorption is less. One way to increase photon absorption even in thin devices is to form a "detuned" resonant cavity where one wall of the cavity is the micromechanical photon detector.

\section{DISCUSSION}

MEMS devices allow us to take advantage of a number of physical phenomena that manifest themselves in the microscale and use such phenomena in the problem of uncooled IR sensing. The approach we presented here relies on the direct interaction of photons with single crystal semiconductor microstructures. For appropriate photon energies such interactions are manifested as internal mechanical stress which cause the microstructures to respond to incoming photons by changing their radius of curvature. An essential aspect of any scheme for micromechanical photon detection is the ability to sensitively detect physical changes resulting from photo-induced stress, since this directly affects the sensitivity and precision in measurement of photon flux. Therefore, sensitive and elegant readout schemes are necessary especially those that can be implemented in large array formats.

Exposing Si microstructures to photons with energies above the bandgap of $\mathrm{Si}$, produces a photo-induced mechanical stress which dominates over thermally-induced stresses. However, when the photon energy is below the Si bandgap thermal effects dominate. We found that the deflection of a Si microstructure depends linearly on photo-induced stress which, in turn, depends linearly on the input optical power and is manifested with fast response times. Furthermore, this mechanism does not rely on changes in the temperature of the microstructure and therefore thermal isolation (that is crucial to the operation of thermal detectors) has minimal influence. Utilizing such a detection mechanism it may be possible to construct micromechanical photon detectors and unlike thermal detectors that respond slowly (response times $\sim \mathrm{ms}$ ) to impinging photons via temperature changes, a detector based on the photo-induced stress will respond both more sensitively and rapidly to incoming photons, with fast response times $<\mathrm{ms}$.

From our present results it can be seen that for $\mathrm{Si}$ microstructures the calculated deflection sensitivity (for $780 \mathrm{~nm}$ photons) is $\Re \sim 0.5 \mathrm{~m} / \mathrm{W}$ and is larger than our measured $\Re=0.099 \mathrm{~m} / \mathrm{W}$. We attribute that difference to the approximations used to determine the photo-induced stress (Eqn 7) and possibly to the value used for the absorbivity of $\mathrm{Si}$. We should note that the smallest microstructure deflection we were able to measure using an optical readout technique was $10^{-11} \mathrm{~m}$. However, values of $10^{-12} \mathrm{~m}$ are possible corresponding to a minimum detectable power of $10^{-11} \mathrm{~W}$ which corresponds to detectivity of $D^{*} \sim$ $10^{9} \mathrm{~cm} \mathrm{~Hz}^{1 / 2} \mathrm{~W}^{-1}$ at $30 \mathrm{~Hz}$. This value is one order of magnitude higher than the $D^{*}$ reported earlier for microcantilever thermal 
detectors ${ }^{14}$. InSb devices have longer cutoff wavelengths and exhibited larger deflection sensitivities which is a direct consequence of its material properties. Indeed, the value for $d \varepsilon_{g} / d P$ is one of the largest compared to other semiconductors.

In order to understand the ultimate performance of MEMS IR detectors we need to consider the fundamental noise sources. At any instant the detector element radiates energy to its surroundings and the surroundings radiate energy to the detector. The temporal fluctuations in this interchange give rise to temporal fluctuations in the temperature of the detector element. These temperature fluctuations appear as fluctuations in the output from the detector element, i.e., as noise. In the microstructure the temperature fluctuations manifest themselves as extremely small motions of the microstructure end, i.e., as fluctuations in $z$. This is known as the background fluctuation limit. No uncooled detector exists today that can operate at the background fluctuation noise limit. The temperature fluctuation noise limit is the fluctuations in temperature of the detector element due to fluctuations in heat exchange with its surroundings by means of thermal conduction. If the micromechanical structures are not in an evacuated package, this dominant heat loss mechanism will probably be caused by thermal conduction through the air surrounding the micromechanical structures. The temperature fluctuation noise associated with conduction through the air will severely limit the performance of the micromechanical structures. Even if the micromechanical structure and the substrate to which it is mounted are at exactly the same temperature, there will be a heat exchange in both directions along the legs which will cause the microstructure temperature to fluctuate; this is known as temperature fluctuation noise. It can be seen that background fluctuation noise is the ultimate manifestation of temperature fluctuation noise; the thermal isolation provided by the legs is so great that radiative losses dominate.

\section{CONCLUSIONS}

IR imaging using uncooled IR detectors is very attractive due to the potential low cost. For efficient IR imaging two dimensional arrays are required and therefore in future work the fabrication of large arrays with small detector elements should be addressed. The results of the present work demonstrate that microstructures represent an important development in MEMS quantum detector technology and can be expected to provide the basis for considerable further development. For example, vastly improved microstructure photon detectors could be produced by making relatively simple changes in the semiconductor material and geometry used in microfabrication. Microstructures can be fabricated using standard semiconductor methods and materials and as a consequence could be mass produced at very low cost. Hence, two-dimensional micromechanical arrays based on the technology described here, could become very attractive for a number of applications due to their inherent simplicity, high sensitivity, and rapid response to optical radiation. In addition, microstructure technology is compatibility with a variety of readout methods also affords tremendous flexibility to potential system designers. The ultimate performance of such devices needs to be further investigated especially when the noise contribution from the readout scheme is considered. Uncooled IR sensor technology can make possible a number of new applications previously not feasible. Although currently uncooled sensors cannot reach the performance characteristics of cooled devices, uncooled detectors with high-density arrays, smaller detector element sizes, low-cost and increased performance will eventually allow us to replace cooled infrared detectors.

\section{ACKNOWLEDGMENTS}

This work was supported by DARPA, and the National Science Foundation. Oak Ridge National Laboratory is operated for the U.S. Department of Energy by Lockheed Martin Energy Research Corporation under contract DE-AC05-960R22464. The author would also like to thank Slo Rajic, Boyd Evans and Joe Cunningham for their contribution.

\section{REFERENCES}

J. L. Miller, Principles of Infrared Technology (Van Nostram Reinhold, New York, 1994).

R. J. Kayes, Optical and Infrared Detectors, Vol. 19 (Springer-Verlag, Berlin, 1977).

E. L. Dereniak and D. G. Crowe, Optical Radiation Detectors (John Wiley \& Sons, 1984).

E. L. Dereniak and G. D. Boreman, Infrared Detectors and Systems (Wiley and Sons, New York, 1996).

A. Rogalski, "New Trends in Infrared Detector Technology," Infrared Physics and Technology 35, 1 (1994).

6

C. Hanson, "Uncooled Thermal Imaging at Texas Instruments," Infrared Technology XXI, SPIE 2020, 330 (1993). 
R. A. Wood, "Uncooled Thermal Imaging with Monolithic Silicon Focal Planes," Infrared Technology XXI, SPIE 2020, 322 (1993).

R. A. Wood and N. A. Foss, "Micromechanical Bolometer Arrays Achieve Low-Cost Imaging," Laser Focus World June, 101 (1993).

P. W. Kruse, "Uncooled IR Focal Plane Arrays," Infrared Technology XXI, SPIE 2552, 556 (1995). for Dual Use," Infrared Technology XXI, SPIE 2552, 583 (1995).

P. G. Datskos, P. I. Oden, T. Thundat, E. A. Wachter, R. J. Warmack, and S. R. Hunter, "Remote Infrared Detection Using Piezoresistive Microcantilevers,” Applied Physics Letters 69, 2986 (1996).

P. I. Oden, E.A. Wachter, P.G. Datskos, T. Thundat, and R.J. Warme
Microcantilevers," SPIE - Infrared Technology XXII 2744, 345 (1996).

P. I. Oden, P. G. Datskos, T. Thundat, and R. J. Warmack, "Uncooled Thermal Imaging Using a Piezoresistive Microcantilevers," Applied Physics Letters 69, 3277 (1996).

E. A. Wachter, T. Thundat, P. G. Datskos, P. I. Oden, S. L. Sharp, and R. J. Warmack, "Remote Optical Detection Using Microcantilevers," Review of Scientific Instruments 67, 3434 (1996).

S. Rajic, B. M. I. Evans, P. G. Datskos, P. I. Oden, G. Thundat, and C. M. Egert, "Piezoresistive Microcantilever Optimization for Uncooled Infrared Detection Technology," Infrared Spaceborne Remote Sensing IV, SPIE 2817, 179 (1996).

\section{R. Amantea, C. M. Knoedler, F. P. Pantuso,
5 mK NEDT, Orlando, FL, 1997, p. 210.}

M. Mao, T. Perazzo, O. Kwon, Y. Zhao, A. Majumdar, J. Varesi, and P. Norton, in Infrared Vision Using an Uncooled Thermo-opto-mechanical Camera; Design, Microfabrication, and Performance, Nashville, TN, 1999 (The American Society of Mechanical Engineers), p. 309.

18 T. Perazzo, M. Mao, O. Kwon, A. Majumdar, J. B. Varesi, and P. Norton, "Infrared Vision Using Uncooled MicroOptomechanical Camera," Applied Physics Letters 74, 3567 (1999).

W. Radford, R. Wyles, J. Wyles, J. Varesi, M. Ray, D. Murphy, A. Kennedy, A. Finch, E. Moody, F. Cheung, R. Coda, and S. Baur, "Microbolometer Uncooled Infrared Camera with $20 \mathrm{mK}$ NETD," Infrared Technology and Applications XXIV 3436, 636 (1998).

W. Radford, D. Murphy, A. Finch, K. Hay, A. Kennedy, M. Ray, A. Sayed, J. Wyldes, R. Wyldes, J. Varesi, E. Moody, and F. Cheung, "Sensitivity Improvements in Uncooled Microbolometer FPAs," Infrared Technology and Applications XXV, SPIE 3698, 119 (1999).

21 J. F. Belcher, C. M. Hanson, H. R. Beratan, K. R. Udayakumar, and K. S. Soch, "Uncooled Monolithic Ferroelectric IRFPA Technology," Infrared Technology and Applications XXIV 3436, 611 (1998).

R. Amantea, L. A. Goodman, F. Pantuso, D. J. Sauer, M. Varhese, T. S. Villianni, and L. K. White, "Progress Towards an Uncooled IR Imager With $5 \mathrm{mK}$ NETD," Infrared Technology and Applications XXIV 3436, 647 (1998).

P. G. Datskos, S. Rajic, and I. Datskou, "Photo-Induced Stress in Silicon Microcantilevers," Applied Physics Letters 73, 2319 (1998).

24 P. G. Datskos, S. Rajic, I. Datskou, and C. M. Egert, "Novel Photon Detection Based on Electronically-Induced Stress in Silicon," IR Detectors and Focal Plane Arrays V, SPIE 3978, 173 (1998).

P. G. Datskos, S. Rajic, C. M. Egert, and I. Datskou, "Detection of Infrared Photons Using the Electronic Stress in MetalSemiconductor Interfaces," Infrared Technology and Applications XXV, SPIE 3698, 151 (1999).

D. Sarid, Scanning Force Microscopy (Oxford University Press, New York, 1991).

J. H. Hoh, J. P. Cleveland, J.-P. Prater, and P. K. Hansma, "Quantized Adhesion Detected with Atomic Force Microscope," Journal of the American Chemical Society 114, 4917 (1992).

F. Ohnesorge and G. Binning, "True Atomic Resolution by Atomic Force Microscopy Through Repulsive and Attractive Forces," Science 260, 1451 (1993).

J. Varesi, J. Lai, T. Perazzo, Z. Shi, and A. Majumdar, "Photothermal Measurements at PicoWatt Resolution Using Uncooled Micro-Optomechanical Sensors," Applied Physics Letters 71, 306 (1997).

J.Lai, T. Perazzo, Z. Shi, and A. Majumdar, "Optimization and Performance of High-Resolution Micro-Optomechanical Thermal Sensors," Sensors and Actuators 58, 113 (1997).

J. G. E. Harris, D. D. Awschalom, K. D. Maranowski, and A. C. Gossard, "Fabrication and Characterization of 100-nm Thick GaAs Cantilevers," Review of Scientific Instruments 67, 3591 (1996). 
33 T. Thundat, P. I. Oden, P. G. Datskos, G. Y. Chen, and R. J. Warmack, in Microcantilever Sensors, Oak Ridge Tennessee, 1996.

34 R. M. White, "Generation of Elastic Waves by Transient Surface Heating," Journal of Applied Physics 34, 3559 (1963).

35 H. K. Wickramasinghe, R. C. Bray, V. Jipson, C. F. Quate, and J. R. Salcedo, "Photoacoustics on a Microscopic Scale," Applied Physics Letters 33, 923 (1978).

36 T. Figielski, "Photostriction Effect in Germanium," Physics Status Solidi 1, 306 (1961).

37 R. G. Stearns and G. S. Kino, "Effect of Electronic Strain on Photoacoustic Generation of Silicon," Applied Physics Letters 47, 1048 (1985).

38 P. Hess and A. C. Boccara, Photoacoustic, Photothermal and Photochemical Processes at Surfaces and in Thin Films (Springer-Verlag, Berlin, 1989).

39 P. G. Datskos, S. Rajic, and I. Datskou, "Detection of Photons Using the Electronic Stress in Metal-Semiconductor Interfaces in MEMS devices," Ultramicroscopy (in press).

40 R. P. Feynman, R. B. Leighton, and M. Sands, The Feynman Lectures on Physics, Vol. 2, 13th ed. (Addison-Wesley Publishing Company, Reading, MA, 1964).

41 R. C. Weast, Handbook of Chemistry and Physics, 59th ed. (CRC, Florida, 1972).

42 P. Aigrain and M. Balkanski, Selected Constants of Semiconductors (Pergamon Press, New York, 1961). 